\title{
Morphological and molecular divergence of Rhipicephalus turanicus tick from Albania and China
}

\author{
Hong-Yu Li ${ }^{1}$ - Shan-Shan Zhao ${ }^{1}$ Sándor Hornok ${ }^{2}$ Róbert Farkas ${ }^{2} \cdot$ \\ Li-Ping Guo ${ }^{1}$ Chuang-Fu Chen ${ }^{3} \cdot \operatorname{Ren}-F u \mathrm{Shao}^{4} \cdot \mathrm{Ji}^{-Z h o u ~} \mathrm{Lv}^{5}$ • \\ Yuan-Zhi Wang ${ }^{1}$
}

Received: 17 June 2017/ Accepted: 1 November 2017/Published online: 27 November 2017

(C) The Author(s) 2017. This article is an open access publication

\begin{abstract}
Rhipicephalus turanicus is an important tick species potentially carrying tickborne pathogens. Several tick species have obvious subspecies divergence. However few studies aimed to examine the existence of divergence within $R$. turanicus. Therefore, a detailed morphological and molecular analysis was conducted for comparing $R$. turanicus from the Mediterranean Basin (represented by Albania) and Central Asia (Northwestern China). Altogether 315 adult ticks of R. turanicus (103 from Albania and 212 from China) were morphologically and molecularly analysed. DNA samples were used for mitochondrial $16 S$ rRNA and coxl gene sequences analysis. In addition, as potentially genetic markers, three fragments including partial nad1-16S rRNA, nad2-coxl, coxl-tRNA-Lys, were designed and then phylogenetically analyzed. Based on detailed morphological observations, only basis capituli length:width ratio (females), the length, the width and the length:width ratio of the scutum (males) had differences between $R$. turanicus from China and Albania. Gene divergences of $16 S$ rRNA, coxl, partial nad1-16S rRNA, nad2-coxl and
\end{abstract}

Hong-Yu Li, Shan-Shan Zhao, Sándor Hornok and Róbert Farkas have contributed equally to this work.

Electronic supplementary material The online version of this article (https://doi.org/10.1007/s10493-0170189-8) contains supplementary material, which is available to authorized users.

Yuan-Zhi Wang

wangyuanzhi621@126.com

1 School of Medicine, Shihezi University, Shihezi 832002, Xinjiang Uygur Autonomous Region, People's Republic of China

2 Department of Parasitology and Zoology, University of Veterinary Medicine, Budapest, Hungary

3 College of Animal Science and Technology, Shihezi University, Shihezi 832002, Xinjiang Uygur Autonomous Region, People’s Republic of China

4 Gene Cology Research Centre, Faculty of Science, Health, Education and Engineering, University of the Sunshine Coast, Maroochydore DC, QLD 4556, Australia

5 Institute of Animal Quarantine, Chinese Academy of Inspection and Quarantine, Beijing 100029, People's Republic of China 
cox1-tRNA-Lys from China and Albania ticks were 3.53-4.84, 3.57-4.92, 3.57-4.07, $3.57-4.39$ and $3.18-4.69 \%$, respectively. The evaluated five genetic markers revealed two phylogenetic branches in $R$. turanicus. Obvious differences exist within $R$. turanicus based on morphological and genetic analysis. Three newly designed genetic markers (partial nad1-16S rRNA, nad2-coxl and coxl-tRNA-Lys) in this study may be suitable genetic tools for identification and analysis in $R$. turanicus. Subspecies analysis of $R$. turanicus from other regions of the world should be initiated in the future.

Keywords Rhipicephalus turanicus · Subspecies · Morphology · Phylogeny

\section{Introduction}

Rhipicephalus turanicus is widely distributed in the Mediterranean sub-region, Africa and Asia, infesting a rich variety of domestic and wild hosts such as sheep, cattle, horses, dogs, cats, and Corsican hares, occasionally even human beings (Chochlakis et al. 2014; DantasTorres et al. 2013). It is a potential or competent vector of several categories of tick-borne pathogens such as Rickettsia spp. (Germanakis et al. 2013) among various hosts and negatively impacts agricultural economies globally. While several tick species have valid subspecies around the world, as exemplified by $R$. evertsi (Guglielmone et al. 2014) and Haemaphysalis erinacei (Hornok et al. 2016), few studies aimed to examine the existence of distinction within $R$. turanicus, despite the known uncertainty in its taxonomy (DantasTorres et al. 2013).

Coxl and 16S rRNA genes are well established barcoding genes for the molecular identification and phylogenetic analyses of ticks. Intraspecies sequence divergence within $R$. turanicus from eastern and central parts of China has been investigated (Lv et al. 2014). Intraspecies genetic variation in the $16 S$ rRNA and cytochrome c oxidase subunit I (coxl) target region have been recorded concerning $R$. turanicus in Yining County, Xinjiang Uygur Autonomous Region (XUAR) (Du et al. 2015), where $R$. turanicus was considered one of the dominant tick species (Wang et al. 2015).

Here, based on complete mitochondrial sequence analysis of the members of Rhipicephalus sanguineus complex (with at least 17 species), 3 mitochondrial hypervariable region fragments, namely, partial nad1-16S rRNA gene, partial nad2-coxl gene and partial coxl-tRNA-Lys gene, together with Coxl and $16 S$ rRNA fragments and detailed morphological study were analysed to investigate divergence of $R$. turanicus originated from Albania and Northwestern China.

\section{Material and methods}

\section{Tick collection}

Two hundred and twelve $R$. turanicus (infesting sheep) were collected from seven counties/cities, including Yining, Fukang, Alataw, Pishan, Qira, Yecheng, and Tumxuk. Similarly, one hundred and three $R$. turanicus from Albania (originated from Helmes-Kavaj, Merrge Lezhe, Lakaret Gjirokaster and Librakhol-Goravash regions) were also used for study. Collected locations and partial submitted sequences information see (Additional Table 1). 


\section{Morphological analysis}

Ticks were first identified as $R$. turanicus according to their morphological characteristics based on the work of Walker et al. (2000) and Filippova (1997). Pictures were made and measurements were performed with a Keyence VHX-5000 digital microscope (Osaka, Japan). The same parameters were measured and compared in the case of males ( 92 from China, 53 from Albania) and females (120 from China, 50 from Albania), except for the peritreme process (used only for females). Morphological study included anteriolateral setae (palp article II), anterior setae (palpal article III), inner length (palpal articles II + III), outer length (palpal articles II + III), palpal width (between palpal articles II/III), basis capituli length, basis capituli width, ratio basis capituli length:width, scutum median length, scutum width, ratio scutum length:width, peritreme median length, peritreme width, peritreme process width and peritreme process median length. Structures of females measured are illustrated in Additional Figure 1. Measurements of ticks were compared with Student $t$ test. Mean values were considered significantly different if $P<0.05$ (Additional Table 3).

\section{Molecular analysis}

Total genomic DNA of $315 R$. turanicus (212 from China and 103 from Albania) was extracted from individual specimens using the TIANamp Genomic DNA Kit (TIANGEN, Beijing, China).

All ticks were first evaluated based on $16 \mathrm{~S}$ rDNA and coxl genetic fragments (Black and Piesman 1994; Chen et al. 2014). Moreover, 13 complete mitochondrial genomes of Rhipicephalus spp. available in GenBank were used to analyze their conservative motifs by DNAMAN 6.0 software (Additional Table 2). Three pairs of primers aimed to hypervariable region fragments were designed by Primer Premier 5.0 software, and their cycling conditions were shown in Additional File 1. The 654 bp fragment of N1 (partial nadl-16S rRNA gene), $595 \mathrm{bp}$ fragment of $N 2$ (partial nad2-coxl gene), and $780 \mathrm{bp}$ fragment of $C 1$ (partial coxl-tRNA-Lys gene) were used as novel genetic markers to analyze divergence of $R$. turanicus. Data analysis methods are described in references (Dantas-Torres et al. 2013; Lv et al. 2014). PCR products were purified using the TIANgel Midi Purification Kit (NGEN, Beijing, China), and sequenced by Sangon Biotech (Shanghai, China). The automatic MEGA model selection method (analysis: Maximum Likelihood model selection, substitution type: nucleotide) was applied to choose the appropriate model for phylogenetic analyses. The dataset was resampled 1000 times to generate bootstrap values. Phylogenetic analyses were conducted with the Maximum Likelihood method by using MEGA v.6.0. Outgroups of phylogenetic trees were selected from GenBank. The 75 nucleotide sequences (16S rDNA: KY583065-KY583081; coxl: KY606287-KY606303; N1: KY620098-KY620114; N2: KY626023-KY626039; and C1: KY996824-KY996840) were deposited in the GenBank database.

\section{Results and discussion}

Concerning evaluated morphological parameters, only the ratio basis capituli length:width was significantly different between $R$. turanicus females from China and Albania $(P=0.022)$. Three parameters were significantly different between $R$. turanicus males 
from China and Albania, i.e. the length, the width and the length: width ratio of the scutum $(P=0.0125,0.0018$ and 0.0027 , respectively) (Additional Table 3 ). The observed differences between one parameter of female and three parameters of male $R$. turanicus collected in China and Albania might be partialy interpreted as geographically related intraspecific morphological variations. Similar differences were noted by Filippova (1997) when comparing $R$. turanicus from four geographical regions in Central Asia (Dagestan, Badaj-Tugaj, Taskent, Sumbar).

Sequencing data confirmed the morphological results on the basis of BLASTn analysis of $16 S$ rRNA and coxl gene, as these had 98.83-99.56 and 98.71-99.88\% homology with the GenBank Italian and American sequences of $R$. turanicus. Based on $16 S \mathrm{rDNA}$ sequence data and Italian $R$. turanicus reference sequence (accession number: KC243864) (Dantas-Torres et al. 2013), $315 R$. turanicus ticks indicated two different lineages of $R$. turanicus originated from from northwestern China and Albania, respectively (Fig. 1). The analysis of sequence divergences showed that the $16 \mathrm{~S} r R N A$ sequences from Chinese $R$. turanicus were $0-1.32 \%$ whereas from Albania they were $0-0.44 \%$. Interestingly, the sequence divergences between $R$. turanicus from Albania and China were 3.53-4.84\%. In addition, based on coxl sequence data, two significantly different lineages also appeared in $R$. turanicus ticks (Fig. 2). The analysis of sequence divergences showed that the coxl sequences from Chinese inners, Albania inners and Chinese-Albania interface were $0-0.47$, $0-1.42$ and $3.57-4.92 \%$, respectively. The latter value might inspire us whether subspecies within it or species complex (Dantas-Torres et al. 2013). 16S rRNA gene phylogenetic trees showed two branches were presented in $R$. turanicus. All $R$. turanicus sequences of northwestern China clustered into the same group, which contains $16 S$ rRNA gene

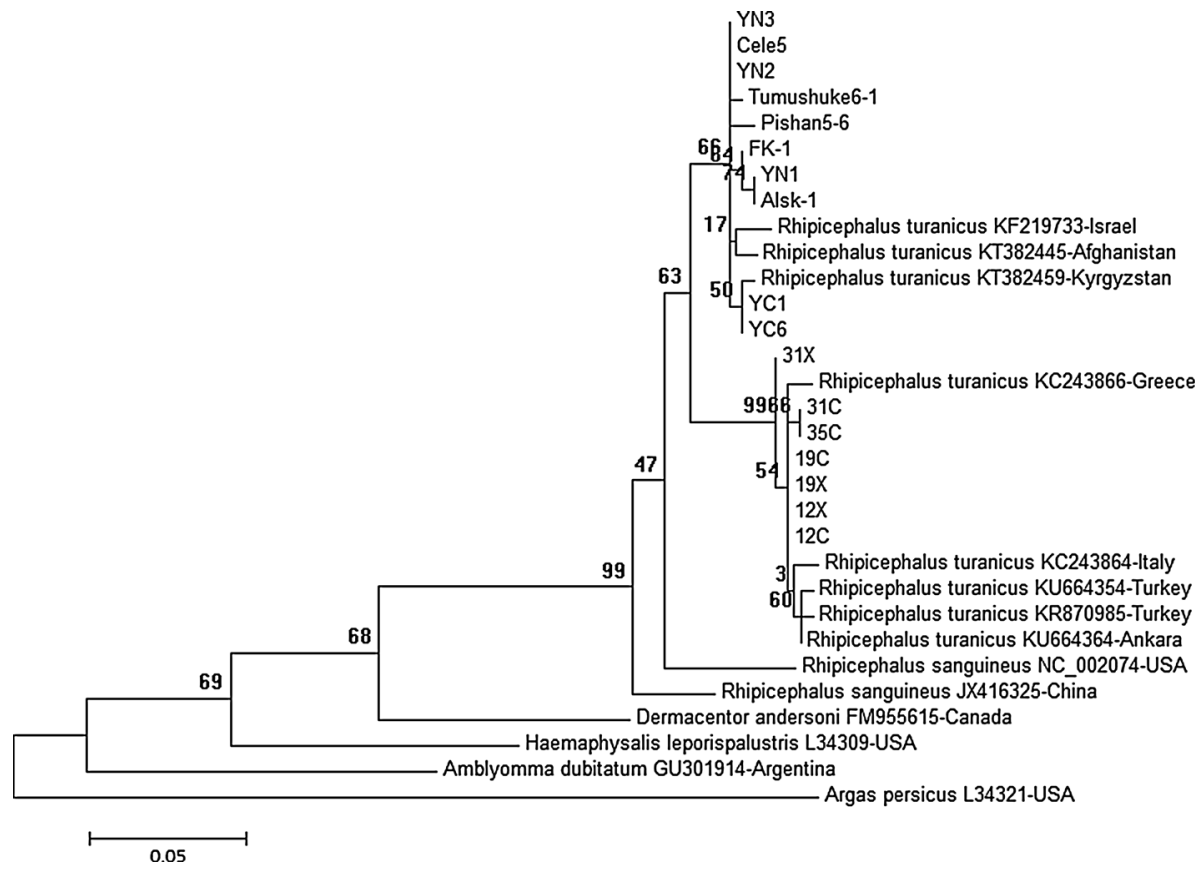

Fig. 1 Phylogenetic comparison of $16 S$ rDNA sequences of Rhipicephalus turanicus. The genotypes of ticks from this study are marked with location and isolate code. Branch lengths represent the number of substitutions per site inferred according to the scale shown 


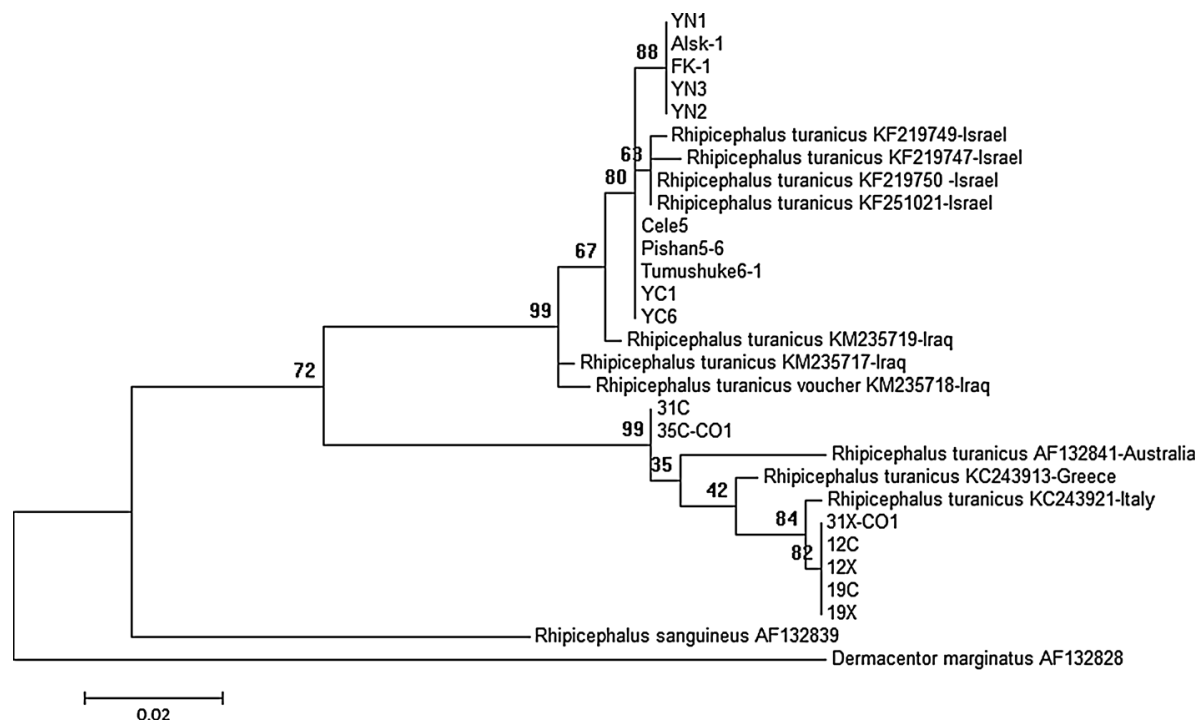

Fig. 2 Phylogenetic relationships of Rhipicephalus turanicus, based on the amplified part of the coxl gene. The genotypes of ticks from this study are marked with location and isolate code. Branch lengths represent the number of substitutions per site inferred according to the scale shown

sequences of Kyrgyzstan (KT382459), Israel (KF219733), and Afghanistan (KT382445), whereas all sequences from Albania clustered into another group combined with sequences from Italy reference sequence (KC243864) (Dantas-Torres et al. 2013), Greece (KC242866), and Turkey (KU664364 and KU664354) (Fig. 1). Similarly, based on coxl phylogenetic analysis, $R$. turanicus sequences of northwestern China were in the same phylogenetic group, which included sequences from Israel (KF219719, KF219747, KF219750 and KF251021) and Iraq (KM235717-KM235719). Meanwhile, together with KC243921 (from Italy reference sequence) (Dantas-Torres et al. 2013), KC243913 (from Greece) and AF132841 (from Australia), the sequences originating from Albania clustered into another branch (Fig. 2).

Three pairs of newly primers aimed to hypervariable mitochondrial region fragments were designed based on 13 complete mitochondrial genomes of Rhipicephalus spp. available in GenBank, which are superior to $16 S$ rRNA and coxl gene owing to their widely coverage than $16 S$ rRNA and coxl gene. When analysing three novel genetic markers ( $N 1, N 2$ and $C 1)$, two different lineages also appeared respectively in $315 R$. turanicus ticks (Fig. 3). The sequence divergences showed that the $\mathrm{N1}, \mathrm{N} 2$ and $\mathrm{Cl}$ sequences from Chinese inners were $0-0.46,0-1.01$ and $0-0.87 \%$, respectively. The divergences from Albania inners were $0-1.22,0-1.27$ and $0-1.67 \%$, while the data from Chinese-Albania interface was 3.57-4.07, 3.57-4.39 and 3.18-4.69\%, respectively. Our three genetic markers of concatenated phylogenetic analysis also support two branches in $R$. turanicus, thus these three genetic markers may be suitable genetic tools for distinction identification analyses (Fig. 3). More ticks originated from other part of the world should be molecularly analyzed by 3 newly markers for the divergence of $R$. turanicus.

In this study, $R$. turanicus originated from northwestern China were collected in semidesert regions, neighbored to Takla makan desert or Gurbantunggut desert. Interestingly, as for geographical characteristics, Afghanistan, Kyrgyzstan and Israel belong to semi-desert 


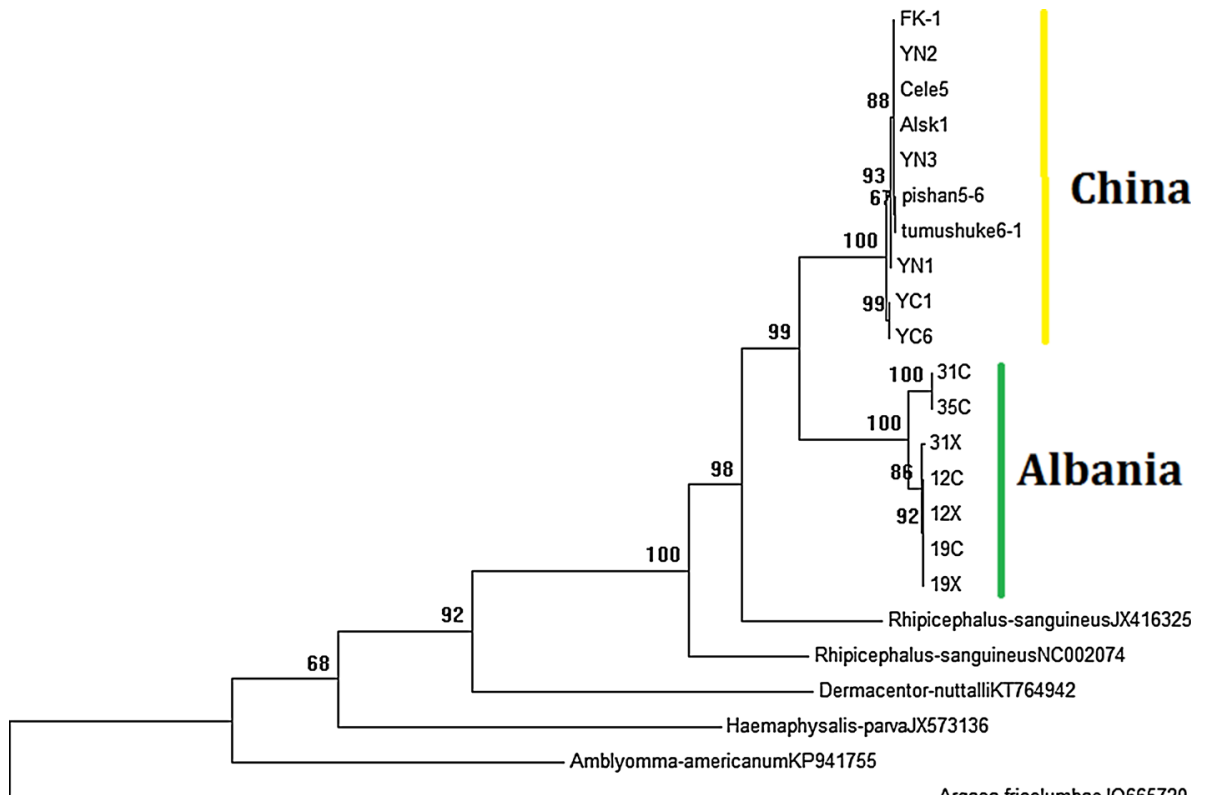

Argasa-fricolumbaeJQ665720

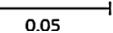

Fig. 3 Phylogenetic tree of the partial N1-N2-C1 concatenated sequence of Rhipicephalus turanicus. The genotypes of ticks from this study are marked with location and isolate code. Branch lengths represent the number of substitutions per site inferred according to the scale shown. The vertical yellow and green lines mark the $R$. turanicus, originated from China and Albania respectively. (Color figure online)

or desert regions, which may support that $R$. turanicus evolved in association with hosts of similar biotopes, although diversification of this lineage was shown based on phylogenetic analysis of $16 \mathrm{~S} r D N A$, coxl and three newly markers data. On the other hand, Albania, Greece and Italy are situated in the Mediterranean Basin, with warm and humid climate in winter and dry and high-temperature in summer, which might have partially contributed to the evolution of different $R$. turanicus lineage compared to northwestern China-originated ones. Accordingly Filipe Dantas-Torres pointed out the existence of different species or taxonomical units within $R$. turanicus (Dantas-Torres et al. 2013).

Overall, morphological, molecular data divergence and phylogenetic trees based on five mitochondrial genetic markers showed that two genetic branches might exist within $R$. turanicus. Phylogenetic analyses generated trees that segregated our tick sequences together with sequences in GenBank, into two distinct clades: one is represented by Central Asia region; the second clade is from the European region, which is in line with former observations by Dantas-Torres on $R$. sanguineus sensu lato from the New and Old worlds (Dantas-Torres et al. 2013). Herein, our findings suggest that $R$. turanicus samples evaluted here from two geographically distant regions showed distinctly divergence. In the future, more efforts are needed to study this, based on a larger number of $R$. turanicus specimens collected throughout its vast geographical range around the world. 


\section{Conclusion}

The present study showed the existence of morphological and molecular divergence among $R$. turanicus (from Albania and China), which needs to be further analysed by larger scale sampling of this species in the Palaearctic. Three novel genetic markers (N1, N2 and C1), in addition to $16 S$ rRNA and coxl gene, may be suitable genetic tools of $R$. turanicus phylogenetic analysis for distinction delineation.

Acknowledgements The authors are grateful to Dr. Jenő Kontschán for providing the digital microscope and for making pictures of $R$. turanicus specimens.

Authors' contributions YZW conceived and designed the study and revised the manuscript. SH performed measurements of $R$. turanicus, and prepared Additional Figure 1. RF provided samples from Albania. HYL wrote the manuscript and SH helped to improve the manuscript. HYL and SSZ performed the laboratory work. LPG, CFC, RFS and JZL collected the ticks. All authors read and approved the final manuscript.

Open Access This article is distributed under the terms of the Creative Commons Attribution 4.0 International License (http://creativecommons.org/licenses/by/4.0/), which permits unrestricted use, distribution, and reproduction in any medium, provided you give appropriate credit to the original author(s) and the source, provide a link to the Creative Commons license, and indicate if changes were made.

\section{References}

Black WC, Piesman J (1994) Phylogeny of hard and soft-tick taxa (Acari: Ixodida) based on mitochondrial $16 S$ rDNA sequences. Proc Natl Acad Sci USA 91:10034-10038

Chen Z, Li Y, Ren Q, Luo J, Liu Z, Zhou X (2014) Dermacentor everestianus hirst, 1926 (Acari: Ixodidae): phylogenetic status inferred from molecular characteristics. Parasitol Res 113(10):3773-3779

Chochlakis D, Ioannou I, Papadopoulos B, Tselentis Y, Psaroulaki A (2014) Rhipicephalus turanicus: from low numbers to complete establishment in Cyprus. Its possible role as a bridge-vector. Parasit Vectors $7: 11$

Dantas-Torres F, Latrofa MS, Annoscia G, Giannelli A, Parisi A, Otranto D (2013) Morphological and genetic diversity of Rhipicephalus sanguineus sensu lato from the New and Old Worlds. Parasit Vectors 6:213

Du J, Mu L, Zhang L, Wang L, Wang A, Zhang K (2015) Morphological classification and molecular identification of in Yining County, Xinjiang Uygur Autonomous Region, China. Chin J Vector Biol Control 26(3):271-274

Filippova NA (1997) Ixodid ticks of subfamily Amblyomminae. Fauna of Russia and neighbouring countries. Nauka Publishing House, St. Petersburg

Germanakis A, Chochlakis D, Angelakis E, Tselentis Y, Psaroulaki A (2013) Rickettsia aeschlimannii infection in a man, Greece. Emerg Infect Dis 19:1176

Guglielmone AA, Robbins RG, Apanaskevich DA, Petney TN, Estrada-Peña A, Horak IG (2014) The hard ticks of the world. Springer, Dordrecht, p 738

Hornok S, Wang Y, Otranto D, Keskin A, Lia RP, Kontschán J (2016) Phylogenetic analysis of Haemaphysalis erinacei Pavesi, 1884 (Acari: Ixodidae) from China, Turkey, Italy and Romania. Parasit Vectors 9:643

Lv J, Wu S, Zhang Y, Chen Y, Feng C, Yuan X (2014) Assessment of four DNA fragments (COI, 16S $r D N A, I T S 2,12 S$ rDNA) for species identification of the Ixodida (Acari: Ixodida). Parasit Vectors 7:93

Walker JB, Keirans JE, Horak IG (2000) Genus Rhipicephalus (Acari, Ixodidae). A guide to the brown ticks of the world. Cambridge University Press, Cambridge

Wang Y, Mu L, Zhang K, Yang M, Zhang L, Du J (2015) A broad-range survey of ticks from livestock in Northern Xinjiang: changes in tick distribution and the isolation of Borrelia burgdorferi sensu stricto. Parasit Vectors 8:449 